\title{
Non-enzymatic oligonucleotide ligation in photoswitchable coacervate protocells sustains compartment-content coupling
}

Tommaso P. Fraccia ${ }^{1, *} \&$ Nicolas Martin ${ }^{2, *}$

1. Institut Pierre-Gilles de Gennes, Chimie Biologie et Innovation, UMR 8231, ESPCI Paris, PSL University, CNRS, 6 rue Jean Calvin, 75005 Paris, France tommaso.fraccia@espci.fr

2. Univ. Bordeaux, CNRS, Centre de Recherche Paul Pascal, UMR 5031, 115 avenue du Dr. Schweitzer, 33600 Pessac, France

nicolas.martin@crpp.cnrs.fr

\begin{abstract}
Modern cells are adaptive chemical compartments tightly regulated by an underlying DNA-encoded program. Reproducing such a coupling between information content and chassis in synthetic compartments represents a key step to the assembly of evolvable protocells, but remains challenging. Here, we rationally exploit complexation between end-reactive oligonucleotides able to stack into long physical polymers and a cationic azobenzene photoswitch to produce three different phases - soft solids, liquid crystalline or isotropic coacervates droplets - that promote non-enzymatic oligonucleotide ligation, with a marked phase-dependent reaction efficiency. Changes in the population of polynucleotides during polymerization induce in turn phase transitions that dramatically alter the physical properties of the compartments. Dynamical modulation of coacervate assembly and dissolution via trans-cis azobenzene photoisomerisation is last used to demonstrate cycles of lightactuated oligonucleotide ligation. Overall, by combining a tight reaction-structure coupling and environmental responsiveness, our light-responsive reactive coacervates provide a novel general route to the non-enzymatic synthesis of polynucleotides, and pave the way to the emergence of a primitive genotype-phenotype coupling in membrane-free protocells.
\end{abstract}

\section{Introduction}

Polynucleotides are essential for life: in modern cells, they act as selective catalysts and signalling molecules, and embody the molecular program underlying genotype-phenotype coupling. Their biosynthesis relies on complex machineries, but the development of chemical routes to their synthesis provides invaluable insights into their abiotic emergence. Pioneering studies have demonstrated nonenzymatic condensation of chemically activated nucleotides using template-directed polymerization, ${ }^{1}$ wet-dry cycles, ${ }^{2}{ }^{3}$ length-selective kinetic trapping in thermal gradients, ${ }^{4}$ adsorption onto mineral surfaces ${ }^{5}$ or accumulation in eutectic ice phases. ${ }^{67}$ Compartmentalization is an attractive alternative approach to concentrate reactive nucleotides, ${ }^{8}$ and offers a higher level of integration, ${ }^{9}$ whereby polynucleotide synthesis could be linked to the macroscopic properties of the compartment itself as a rudimentary form of genotype-phenotype coupling. However, connecting enzyme-free replication of compartmentalized polynucleotides to replication of membrane-bounded protocells remains challenging. ${ }^{9}{ }^{10}$ Coacervate droplets represent a promising platform to demonstrate this connection.

Coacervates produced by associative liquid-liquid phase separation in water have recently emerged as viable alternative protocells. ${ }^{11} 1^{12}{ }^{13}{ }^{14}$ These membrane-free microdroplets form in mixtures of prebiotically relevant species ${ }^{11} 1215$ (including mono- and oligonucleotides, oligopeptides, short polyamines and fatty acids), selectively sequester biomolecular solutes, ${ }^{16}{ }^{17}$ support ribozyme activity and template-directed nucleotide polymerization, ${ }^{18}{ }^{19}$ guides the interfacial assembly of lipid membranes ${ }^{20} 2122$ and can organize into multiple sub-compartments. ${ }^{23}{ }^{24}$ Non-enzymatic templatefree polynucleotide synthesis in coacervates has not yet been reported. Remarkably, the formation and stability of coacervates are intrinsically related to the properties of their scaffold constituents, 
among which the length, flexibility or charge density of polyions, ${ }^{25}{ }^{26}$ making them attractive systems to demonstrate dynamic coupling between molecular content, in situ reactivity and compartment structure. Recent studies have documented the use of reactions to control the formation and dissolution of coacervates with enzymes ${ }^{27} 282930$ or dissipative chemical cycles, ${ }^{31}{ }^{32}$ but the real potential of these systems to selectively couple in situ reactivity with coacervate properties has not yet been realized.

Our strategy relies on oligonucleotides being capable of end-stacking into long liquid crystalline-ordered physical polymers at high concentrations, ${ }^{33}{ }^{34}$ a phenomenon that has been reported to promote their non-enzymatic chemical ligation into longer polynucleotides. ${ }^{35}{ }^{36}$ Liquid crystal (LC) ordering was also recently observed in dilute oligonucleotide solutions upon complex coacervation with an oppositely charged oligopeptide. ${ }^{37}{ }^{38} 39$ Building upon these ordered interactions, we show here that mixtures of end-stacking reactive oligonucleotides and a molecular azobenzene cation produce three different phases ("phenotypes") - soft solids, liquid crystalline or isotropic coacervates droplets - that significantly enhance oligonucleotide ligation, with a marked phasedependent reaction efficiency. Excitingly, changes in the length distribution of polynucleotides during polymerization ("genotype" variation) induce phase transitions that dramatically alter the physical properties of the compartments ("phenotype" modification). Light-mediated trans-cis azobenzene photoisomerisation dynamically modulates the formation and dissolution of coacervate droplets, ${ }^{40} 41$ so that cycles of light-actuated oligonucleotide ligation are eventually achieved to demonstrate temporal control over polynucleotide elongation in periodically changing environmental conditions. Although the design of our system does not solely involve prebiotically relevant components, our complex coacervation platform offers a general approach to the non-enzymatic synthesis of polynucleotides from shorter oligomers by combining a tight reaction-structure relationship and environmental responsiveness, and paves the way to the emergence of compartment-content coupling in membrane-free protocells.

\section{Results and discussion}

\section{Phase behaviour of oligonucleotides and azobenzene cations}

We first investigated the phase behaviour of charge-balanced mixtures of trans-azobenzene trimethylammonium bromide (trans-azoTAB, $5 \mathrm{mM}$, Fig. 1a) and a self-complementary DNA Dickerson dodecamer sequence (DD, 5'-CGCGAATTCGCG-3', $1.5 \mathrm{mg} \mathrm{mL}^{-1}, 5 \mathrm{mM}$ nucleobase concentration), which hybridizes into blunt-end duplexes capable of liquid crystal ordering at high concentration ${ }^{33} 3442$ (> $300 \mathrm{mg} \mathrm{mL}^{-1}$, Fig. 1a). Optical microscopy images revealed the formation of solid-like precipitates in pure water (Supplementary Fig. 1), while soft solids or liquid-like microdroplets were observed when the ionic strength was gradually increased (Fig. 1b and Supplementary Fig. 2). Such a salt-dependent solid-to-liquid transition has already been reported in mixtures of oppositely charged polyelectrolytes, ${ }^{43}$ including oligonucleotides and polypeptides, ${ }^{12} 263739$ and attributed to a competition between polymer rigidity and charge screening for sufficiently long and stiff polyelectrolytes. ${ }^{39}{ }^{44}$ Coacervate microdroplets further disassembled above a critical salt concentration (Fig. 1b and Supplementary Fig. 1, $[\mathrm{NaCl}]>520 \mathrm{mM}$ ), indicating that electrostatic interactions were a driving force for DD/trans-azoTAB complexation.

Strikingly, droplets prepared at intermediate salt concentrations exhibited a strong birefringence under crossed polarisers, with textures typical of columnar liquid crystalline phase ${ }^{37}$ (Fig. 1b, $200 \mathrm{mM}$, and Supplementary Fig. 3), while they appeared isotropic (ISO) at higher ionic strength (Fig. 1b, $350 \mathrm{mM}$ ). These observations were in line with the salt-dependent formation of ISO or LC coacervate droplets recently reported in DD/poly-L-Lysine mixtures, ${ }^{37}$ where LC ordering was attributed to the supramolecular assembly of DD duplexes into long linear aggregates via end-to-end stacking, as previously observed in highly concentrated oligonucleotide solutions. ${ }^{33} 3442$ Here, inhibition of end-to-end interactions by the addition of TT overhangs at the 3 '-terminus of the DD sequence prevented the formation of LC droplets (Supplementary Fig. 4), which confirmed that sufficiently long 
and stiff aggregates were required for LC organisation. ${ }^{37}$ This observation revealed that coacervate phases were intrinsically dependent on the oligonucleotide sequence.

Taken together, our observations suggest that charge-balanced mixtures of a selfcomplementary DNA dodecamer and trans-azoTAB produce ordered LC coacervate microdroplets at intermediate salt concentrations, which is attributed to the physical linear aggregation of blunt-ends oligonucleotides into longer stiff polynucleotides, together with a possible contribution from azobenzene $\pi-\pi$ self-stacking ${ }^{45}$ (Figs. 1a,c). In comparison, an isotropic coacervate phase is produced at higher ionic strength, presumably associated to disordered monomeric or short repeats of DD duplexes bridged together by trans-azoTAB (Fig. 1c). This behaviour is in line with the known concentration dependence for the reversible linear aggregation of end-to-end interacting oligomeric DNA duplexes, which undergoes a sharp discontinuous increase at the ISO-LC transition. ${ }^{46}$

a

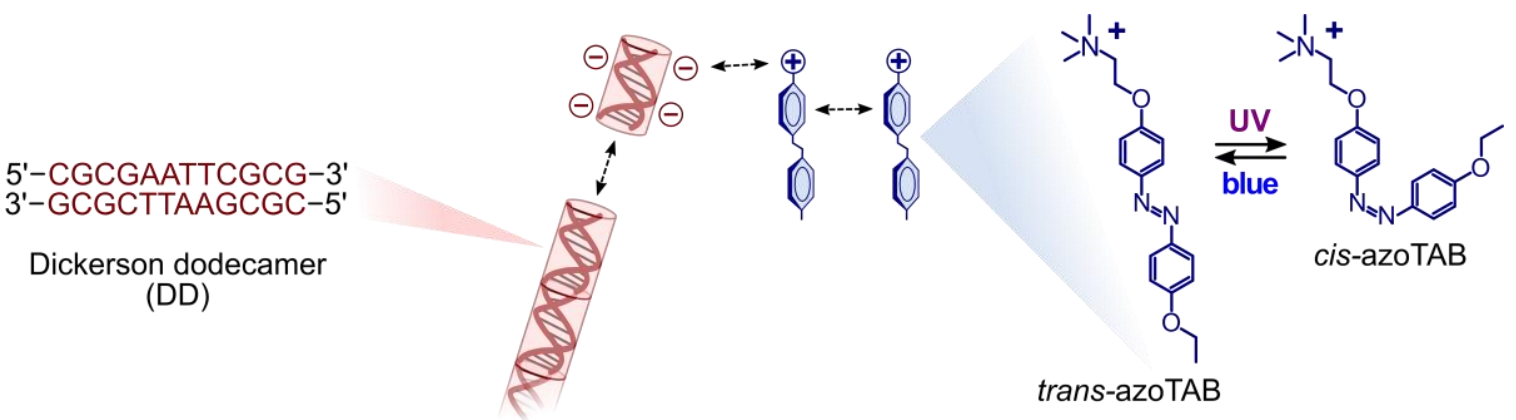

b

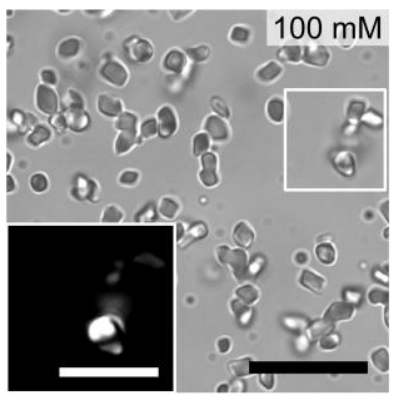

soft solids

C

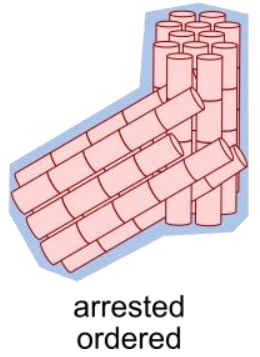

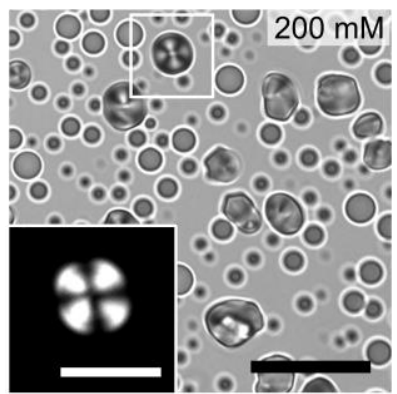

LC coacervates

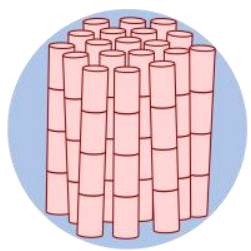

fluid ordered

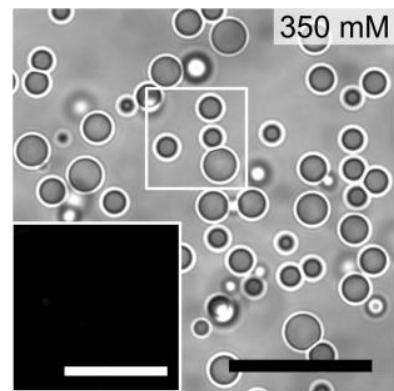

ISO coacervates

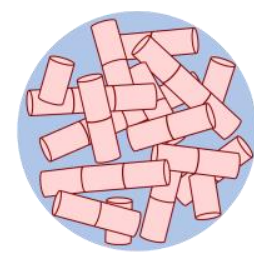

fluid disordered

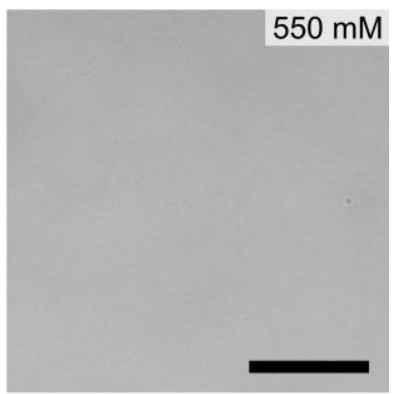

no phase separation

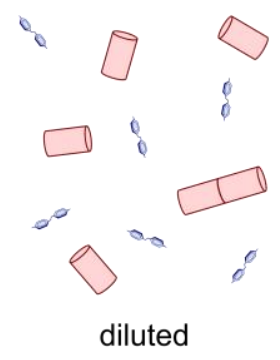

Figure 1. Phase behaviour of end-interacting oligonucleotides and azobenzene cations. a, Sequence and hybridization of the self-complementary DNA Dickerson dodecamer (DD) used in the study (left), chemical structure of trans-azoTAB and reversible photo-isomerisation to cis-azoTAB (right), and schematic representation of interactions involved in DD/trans-azoTAB complexation (middle). b. Bright-field optical microscopy images of charge-balanced mixtures of DD (5 mM nucleobase concentration) and trans-azoTAB (5 $\mathrm{mM}$ ) prepared at different $\mathrm{NaCl}$ concentrations, as indicated, showing the formation of irregular soft solids (100 $\mathrm{mM} \mathrm{NaCl})$, liquid crystal (LC) coacervate droplets $(200 \mathrm{mM} \mathrm{NaCl})$, isotropic (ISO) coacervate droplets ( $350 \mathrm{mM} \mathrm{NaCl})$, or absence of phase separation ( $550 \mathrm{mM} \mathrm{NaCl}$ ). Scale bars, $20 \mu \mathrm{m}$. Insets show zoomed images of the white squared areas under $90^{\circ}$ crossed polarisers. Scale bars, $10 \mu \mathrm{m}$. c, Schematic views of the self-assembled complexes produced at the different salt concentrations shown in $\mathbf{b}$. 


\section{Non-enzymatic oligonucleotide ligation is enhanced in coacervate droplets}

Quantitative measurements of the oligonucleotide concentration in the LC and ISO coacervate droplets revealed that $\sim 90 \mathrm{~mol} \%$ of DD strands were sequestered within the coacervate phase, corresponding to a local oligonucleotide concentration of $\sim 480 \pm 190 \mathrm{mg} \mathrm{mL}^{-1}$ in the ISO microdroplets (Supplementary Note 1). In comparison, the oligonucleotide concentration in the supernatant was $0.72 \pm 0.3 \mathrm{mg} \mathrm{mL}^{-1}$, so that a $\sim 670$-fold enhancement of DD local concentration in the ISO coacervates compared to the supernatant was observed. We sought to utilize this strong enrichment of DNA oligomers within coacervate phases to achieve in situ non-enzymatic oligonucleotide polymerisation. For this purpose, DD strands were functionalized with a $3^{\prime}$ terminal phosphate group to produce endreactive DDp oligonucleotides (5'-CGCGAATTCGCG- $p$ - $3^{\prime}$ ) able to undergo covalent ligation, via a phosphodiester bond, in the presence of the water-soluble coupling agent 1-ethyl-3-(3dimethylaminopropyl) carbodiimide hydrochloride ${ }^{35}$ (EDC, Supplementary Fig. 5). Due to its sensitivity to hydrolysis, a large molar excess of EDC compared to the reactive phosphate groups is typically required. ${ }^{35}{ }^{36}$ Here, the contribution of EDC salt to the total ionic strength of the solution produced a similar solid-to-liquid transition as $\mathrm{NaCl}$, and had therefore to be taken into account to produce the desired oligonucleotide/trans-azoTAB assemblies (Supplementary Fig. 6).

The outcome of the ligation reaction was first monitored after 24 hours in three representative $\mathrm{DDp} /$ trans-azoTAB samples prepared at varying $\mathrm{NaCl}$ but fixed EDC concentrations (soft solids: $80 \mathrm{mM}$ EDC; LC coacervates: $100 \mathrm{mM} \mathrm{NaCl}+80 \mathrm{mM}$ EDC; ISO coacervates: $200 \mathrm{mM} \mathrm{NaCl}+80 \mathrm{mM} \mathrm{EDC}$ ) using polyacrylamide gel electrophoresis (PAGE), and compared to the supernatant solution. These observations revealed that oligonucleotide ligation occurred efficiently in DDp-rich phases (soft solids and coacervates), as manifested by the appearance of a large distribution of oligomer bands in the polyacrylamide gels, but was very limited in the dilute supernatant phase (Fig. 2a and Supplementary Fig. 7). Notably, the maximum degree of polymerisation, $n_{\max }$, observed in the gels was significantly higher in DDp-rich phases ( $n_{\max }$, soft solids $=9 ; n_{\max }$ LC droplets $=12 ; n_{\max }$, ISO droplets $=6$ ) compared to the dilute supernatant solution $\left(n_{\max }\right.$, supernatant $\left.=2\right)$. More extensive investigations confirmed that ligation was also ineffective in samples prepared at higher salt concentrations (Supplementary Fig. 8), as expected from the lower coacervate volume phase produced near the critical coacervation salt concentration. We attributed such differences in reaction efficiency mainly to differences in the nature of DDp/transazoTAB phases, since $\mathrm{NaCl}$ addition has been shown to have minimal effect on EDC-activated DNA ligation. ${ }^{47}$

Fluorescence intensity profiles of the gel scans were analysed more quantitatively by exploiting the approximate proportionality between electrophoretic mobility and logarithm of chain length ${ }^{35} 48$ (Fig. 2b). The cumulative weight fraction distribution, $C(n)$, extracted from these profiles was well described by a simple Flory model for linear polymer condensation ${ }^{49}$ (Fig. $\mathbf{2 b}$ and Supplementary Note 2), despite uncertainties in the quantitative information carried by the fluorescence profiles of the gels. This model allowed us to quantify and compare the polymerization yield, $p$, and the mean degree of polymerization, $\langle n\rangle$, for the different conditions tested (Supplementary Note 3). We observed that both the polymerization yield and average degree of polymerization were the highest for LC coacervate droplets $(p=0.55 \pm 0.01,\langle n\rangle=3.40 \pm 0.10)$, then decreased for soft solids $(p=0.47 \pm 0.01,\langle n\rangle=2.77 \pm 0.07)$ and ISO coacervate droplets $(p=0.29 \pm$ $0.01,\langle n\rangle=1.82 \pm 0.04)$, and were the lowest for the supernatant solution $(p=0.06 \pm 0.01,\langle n\rangle=$ $1.13 \pm 0.02$ ). The same tendency for $p$ and $\langle n\rangle$ was observed at different reaction times (Fig. $2 \mathrm{c}$ and Supplementary Fig. 9). The characteristic reaction time, $\tau$, extracted from fitting of $p$ vs. time using a catalysed step-growth polymerization model (Supplementary Note 4) was $9.0 \pm 0.7$ h, $4.5 \pm 0.9$ h, 22 $\pm 4 \mathrm{~h}$ and $227 \mathrm{~h}$ for soft solids, LC coacervates, ISO droplets and supernatant, respectively, corresponding to a 25 - or 50- or 10 -fold acceleration in soft solids, LC coacervates or ISO droplets, respectively, compared to the supernatant. In addition, the products distribution gradually moved toward longer DNA sequences as a function of time while the fraction of unreacted DDp oligonucleotides $(n=1)$ decreased (Supplementary Fig. 10), so that different DNA populations were obtained after 24 hours in the different DDp-rich phases (Fig. 2d). 

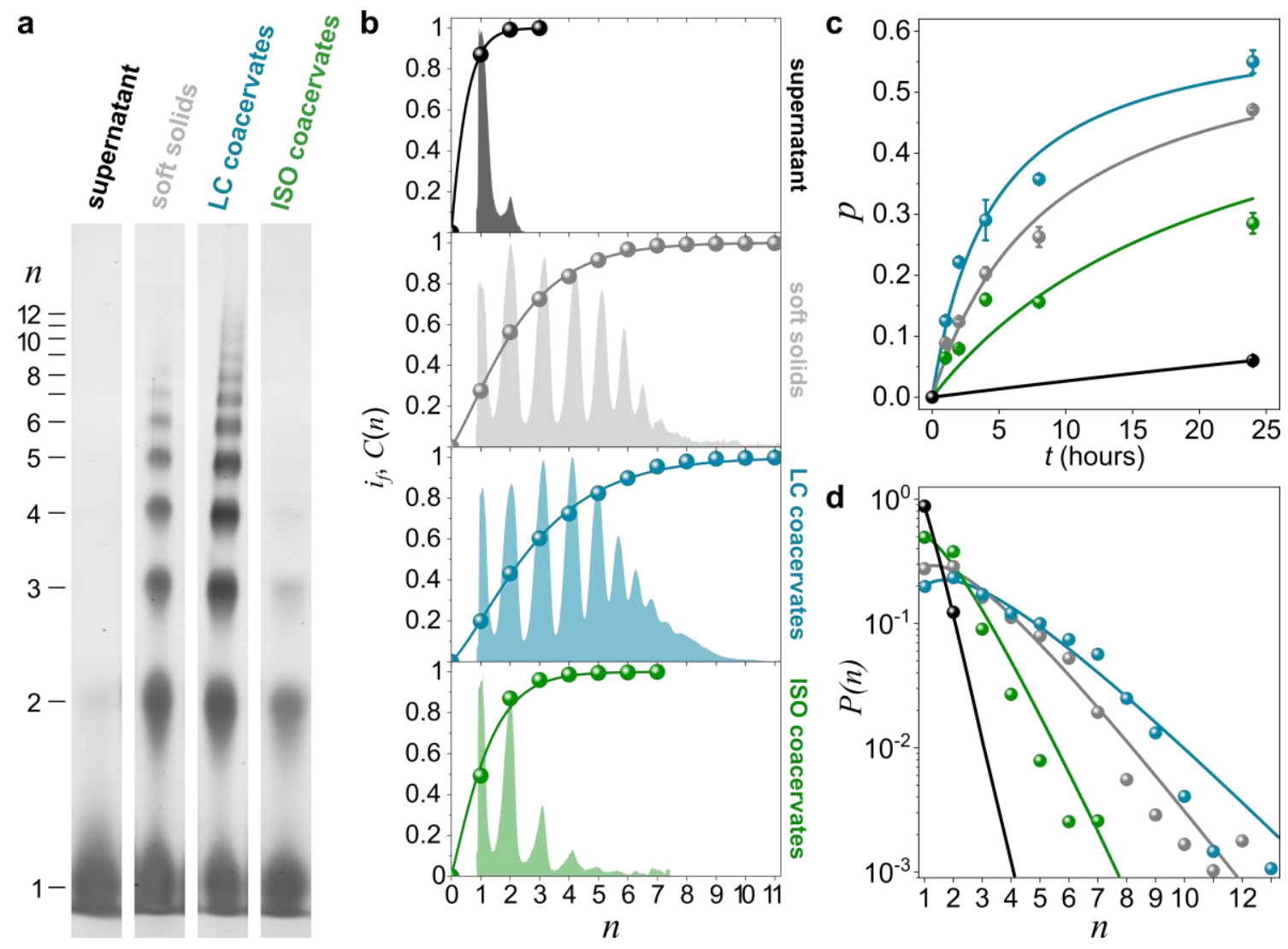

Figure 2. Oligonucleotide ligation depends on the nature of the DDp/trans-azoTAB phase. a, Cropped gel images of a 15\% PAGE run of the ligation product obtained after 24 hours DDp/trans-azoTAB solutions (equimolar charge mixtures, $10 \mathrm{mM}$ total charge concentration) in different phases, as labelled (soft solids: $80 \mathrm{mM}$ EDC; LC droplets: $100 \mathrm{mM} \mathrm{NaCl}+80 \mathrm{mM} \mathrm{EDC}$; ISO droplets: $200 \mathrm{mM} \mathrm{NaCl}+80 \mathrm{mM}$ EDC; supernatant obtained from centrifuged LC droplets). Full gel images are shown on Supplementary Fig. 7. b, Fluorescence intensity $\left(i_{f}\right.$, colored areas), extracted from gel images shown in a (i.e., after 24 hours of reaction) and cumulative weight distribution $(C(n)$, colored dots) plotted as a function of degree of polymerization, $n$, derived from rescaling (see Supplementary Note 3). Solid lines show fits of $C(n)$ data points using Flory's theory for linear polymer condensation (see Supplementary Note 2). c, Time-dependent evolution of polymerization yield, $p$, for the different solutions prepared as in a (black: supernatant, grey: soft solids, cyan: LC coacervates, green: ISO coacervates). Solid lines are fits using a catalysed step-growth polymerization model to estimate the characteristic reaction time, $\tau$ (see Supplementary Note 4). Values were extracted by processing time-dependent PAGE scans shown in Supplementary Fig. 7 as in b. Error bars represent errors associated to uncertainties in the fits used to extract the reaction yield (see Supplementary Note 3). d, Weight fraction distributions, $P(n)$, after 24 hours of ligation reaction in the different solutions prepared as in a (black: supernatant, grey: soft solids, cyan: LC coacervates, green: ISO coacervates). Solid lines show fits of $P(n)$ data points using Flory's theory for linear polymer condensation (see Supplementary Note 2).

Overall, these analyses confirmed that ligation was most enhanced in LC coacervates, followed by soft solids and ISO droplets. The enhanced reaction efficiency in the dense complexes compared to the supernatant was attributed to the higher local concentration of oligonucleotides in the condensates. Additional mechanisms contributing to the increased ligation efficiency in LC coacervates compared to soft solids or ISO droplets included stabilization of end-to-end supramolecular association of DNA oligonucleotides into organized repeats characteristic of the already ligated bases ${ }^{46} 50$ maintenance of stable proximity of the reacting termini, ${ }^{51}$ and provision by the LC phase of a still fluid environment for transport and reaction. ${ }^{36}$ Importantly, reaction yields in LC droplets were constantly higher than those observed in soft solids (Fig. 2c), confirming that the suppressed fluidity in the latter affected EDC diffusion and DDp reorganization, in turn reducing ligation efficiency, despite the likely higher concentration of oligonucleotides in such low-salt phase (Supplementary Note 1). 


\section{Oligonucleotide elongation produces phase transitions and multiphase coacervate droplets}

Samples were further observed before and after the 24 hour ligation reaction. Optical microscopy images revealed that soft solids and LC coacervate droplets evolved towards crystalline-like assemblies after the reaction (Figs. $\mathbf{3 a}, \mathbf{b}$ ), which we attributed to the formation of long covalent polynucleotides that produced less fluid, more organized complexes. ${ }^{33}$ More strikingly, ISO coacervates exhibited a multiphase droplet organization after ligation, as revealed by the presence of a newly formed birefringent coacervate sub-phase (Fig. 3c). Droplets doped with a DNA intercalating dye further showed a brighter fluorescence within this phase compared to the surrounding coacervate layer (Fig. 3d and Supplementary Fig. 11), suggesting that the inner phase contained a higher nucleotide density. Time-dependent experiments further pointed to the gradual growth of this coacervate sub-phase over time (Supplementary Fig. 12), which correlated with the gradual advancement of the ligation reaction. After 96 hours, most of the droplets became completely birefringent and less spherical, with typical textures of columnar LC. In comparison, control experiments using non-reactive DD oligonucleotides did not undergo structural change over time (Supplementary Fig. 12).

a
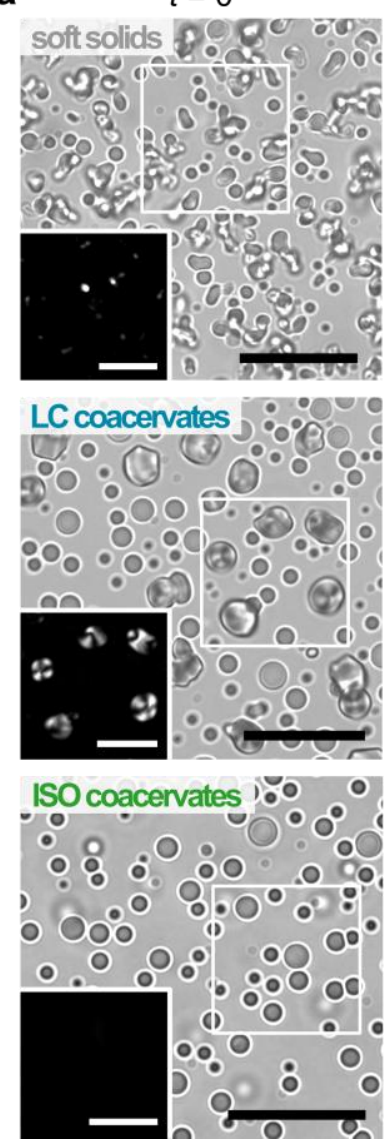
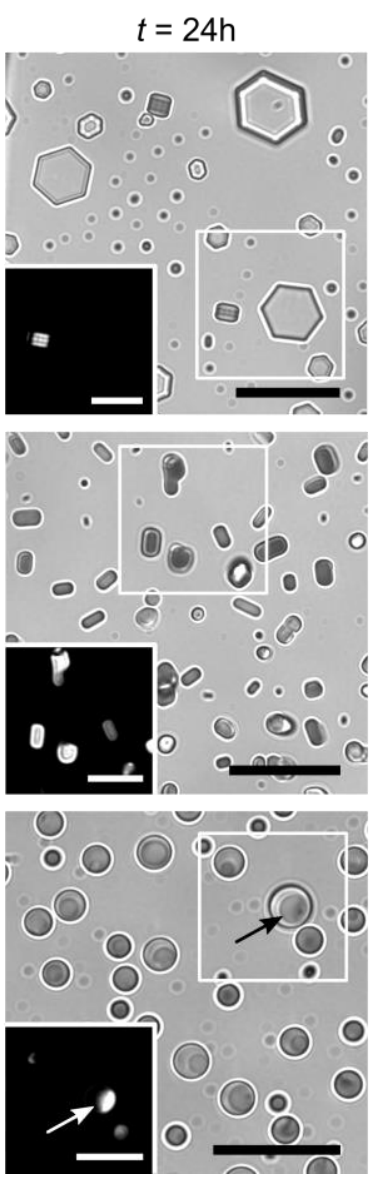

b

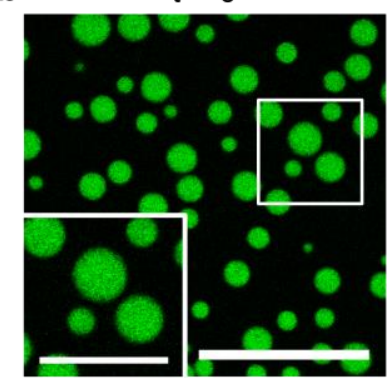

C

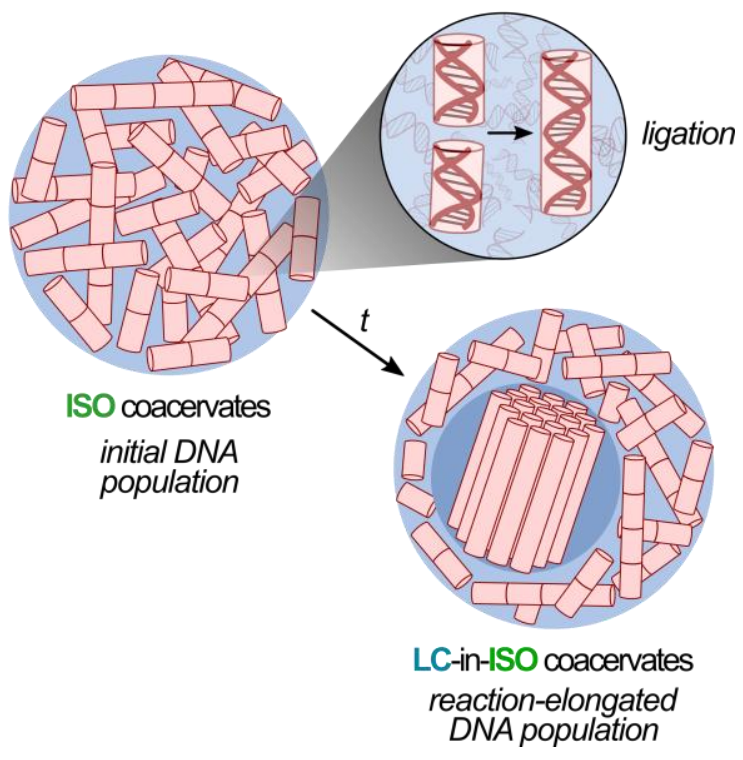

Figure 3. Structural transformation of coacervates during oligonucleotide ligation. a, Bright-field optical microscopy images of charged-balanced reactive mixtures of DDp ( $5 \mathrm{mM}$ nucleobase concentration) and transazoTAB (5 mM) prepared at different total ionic strength (soft solids: $80 \mathrm{mM}$ EDC (top); LC droplets: $100 \mathrm{mM}$ $\mathrm{NaCl}+80 \mathrm{mM} \mathrm{EDC} \mathrm{(middle);} \mathrm{ISO} \mathrm{droplets:} 200 \mathrm{mM} \mathrm{NaCl}+80 \mathrm{mM} \mathrm{EDC} \mathrm{(bottom))} \mathrm{at} t=0$ and after 24 hours, as labelled. Scale bars, $20 \mu \mathrm{m}$. Insets show the white squared areas under $90^{\circ}$ crossed polarisers. Scale bars, $10 \mu \mathrm{m}$. b, Confocal fluorescence microscopy images of reactive ISO coacervate droplets (5 mM DDp nucleobase concentration, $5 \mathrm{mM}$ trans-azoTAB, $200 \mathrm{mM} \mathrm{NaCl}+80 \mathrm{mM} \mathrm{EDC}$ ) doped with $1 \times$ SYBR Gold at $t=0$ and after 24 hours of reaction. Scale bars, $20 \mu \mathrm{m}$. Insets show zoomed images of the white squared areas. Scale bars, $10 \mu \mathrm{m}$. c, Schematic representation of the transformation of ISO coacervate droplets into multiphase LC-in-ISO coacervate droplets during ligation. 
These observations suggested that the coexistence of ISO and LC phases in coacervate droplets was driven by the gradual enrichment of the DNA population in long polynucleotides produced by DDp ligation, and their preferential partitioning in the columnar sub-phase (Fig. 3c). Such behaviour is consistent with the higher degree of order expected for long polynucleotides chains compared to shorter ones s $^{33442}$ and with spontaneous phase separations observed in highly polydisperse LC forming DNA solutions. ${ }^{52}$ This hypothesis was corroborated by the observation that boundaries in the phase diagram of trans-azoTAB and the self-complementary 24-mer oligonucleotide $\mathrm{DD}_{2}$ (in which the $\mathrm{DD}$ sequence was repeated: 5'-CGCGAATTCGCGCGCGAATTCGCG-3') were shifted to higher ionic strength compared to DD (Fig. 4a), so that LC coacervates rather than ISO droplets were produced at $350 \mathrm{mM}$ $\mathrm{NaCl}$ for $\mathrm{DD}_{2} /$ trans-azoTAB, and a higher critical coacervation salt concentration was observed compared to DD/trans-azoTAB. Length-dependent phase transitions are peculiar of the LC nature of this coacervate system, since longer DNA duplexes order at lower concentration, ${ }^{46}$ while the increase in coacervate stability with polymer length is well established, ${ }^{25}$ and has been observed in oligonucleotide coacervates. ${ }^{26} 39$ In addition, multiphase coacervation has been shown to occur when two coacervate systems with sufficiently different critical coacervation salt concentrations are mixed together at low ionic strength, a condition under which droplets with a sufficient difference in surface tension are formed. ${ }^{23}{ }^{24}$ Accordingly, mixtures of DD, $\mathrm{DD}_{2}$ and trans-azoTAB produced multiphase LCin-ISO coacervate droplets containing birefringent columnar sub-domains at $350 \mathrm{mM} \mathrm{NaCl}$ (Supplementary Fig. 13), a condition at which DD alone formed uniformly isotropic coacervate droplets.

\section{Oligonucleotide ligation in photoswitchable coacervates can be reversibly controlled with light}

An additional feature that makes coacervates droplets relevant protocellular models is their strong responsiveness to environmental parameters, such as salinity, temperature and $\mathrm{pH}$, that could conceivably periodically vary in prebiotic environments subjected to daily and seasonal hydration and temperature cycles. In such a scenario, coacervates dissolution and reassembly can regulate reactivity and provide selection steps. Since temperature and hydration variations may affect condensation rates, ${ }^{47}$ we sought to isolate the contribution of reversible coacervation cycles by exploiting light as an orthogonal stimulus, and demonstrate reversible photocontrol over abiotic oligonucleotide ligation based on azoTAB trans-cis photoisomerization. When exposed to UV light, trans-azoTAB quantitatively isomerizes into cis-azoTAB, which decreases its binding affinity to double-stranded DNA (dsDNA), ${ }^{40} 53$ 545566 a process that can be partially reversed with visible light (Supplementary Fig. 14). This strategy has been used recently to design DNA coacervate droplets that are formed and dissolved in response to light. ${ }^{40}$ Here we aimed to exploit such a photoswitchable behaviour to regulate the ligation reaction based on the dilution/concentration of oligonucleotides associated to the dissolution/condensation of coacervate droplets.

Equilibrium phase behaviour studies of DD at varying trans:cis-azoTAB molar fractions revealed that lower fractions of trans-azoTAB favoured the formation of liquid-like ISO coacervates at lower ionic strength, and resulted in lower critical salt concentrations for coacervate dissolution (Fig. 4a and Supplementary Fig. 15). These observations confirmed that cis-azoTAB had a lower binding affinity for $D D$ compared to trans-azoTAB, which opened the possibility to navigate across the DD/azoTAB phases at fixed salt concentration using light (Fig. 4a). Notably, LC and ISO coacervate microdroplets readily dissolved in a few seconds when exposed to UV light due to quantitative transcis azoTAB photoisomerisation, and reassembled into ISO droplets under blue light due to the reverse cis-trans azoTAB photoisomerisation (Fig.4b and Supplementary Fig. 16). This process was reversible and could be repeated several times without any apparent fatigue (Supplementary Fig. 17). In comparison, salt-free solid-like precipitates transformed into liquid-like ISO coacervate droplets under UV light but did not dissolve (Supplementary Fig. 16), as expected from the equilibrium phase diagram and similar to recently reported photo-controllable phase transitions in arylazopyrazole-conjugated oligonucleotide coacervates. ${ }^{57}$ 
a

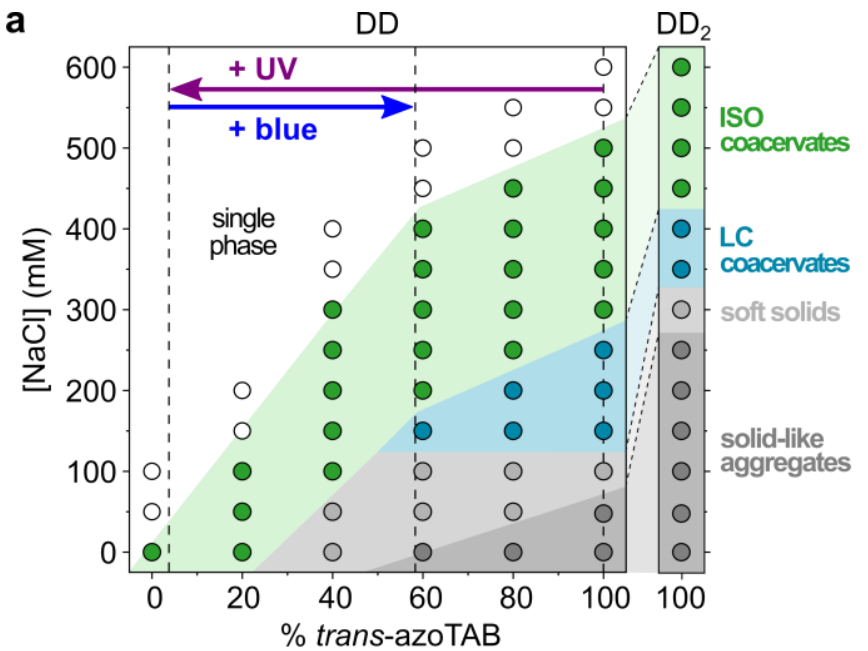

+ UV

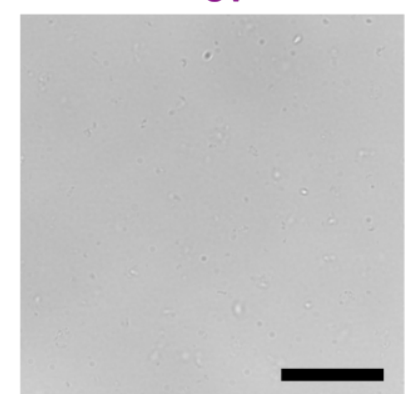

C

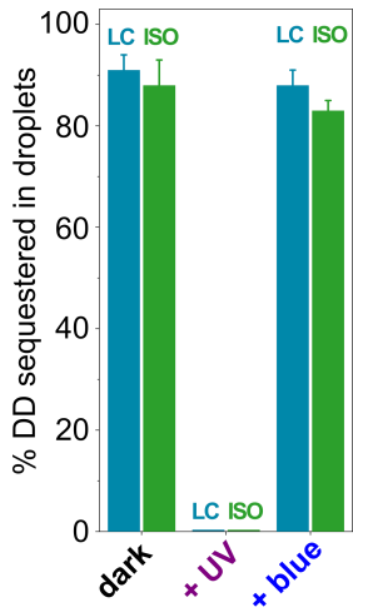

b

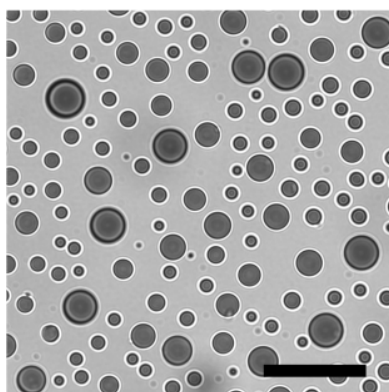

d $0 . 6 \longdiv { \text { LC coacervates } }$

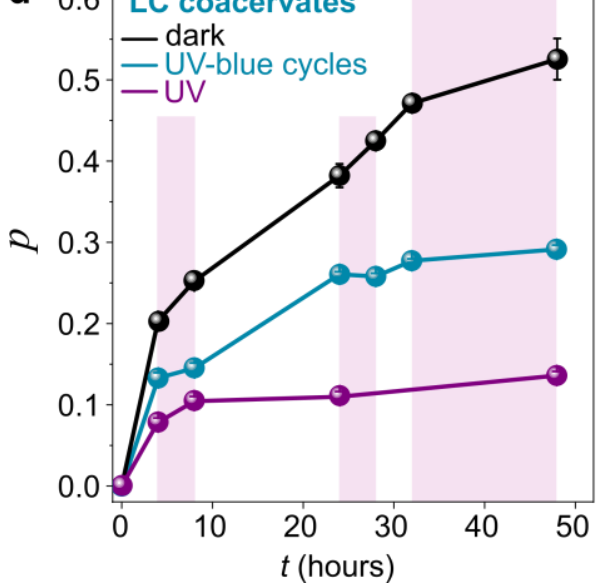

Figure 4. Light-modulated phase separation and oligonucleotide ligation. a, Equilibrium phase diagram of charge-balanced mixtures of $\mathrm{DD}$ or $\mathrm{DD}_{2}$ (5 mM nucleobase concentration) and azoTAB (5 mM total concentration) at varying trans:cis fraction or at $100 \%$ trans-azoTAB for $\mathrm{DD}$ or $\mathrm{DD}_{2}$, respectively, and at varying $\mathrm{NaCl}$ concentration. The complexes produced for each condition were characterized by optical microscopy (as shown in Supplementary Fig. 15) and classified as solid-like aggregates (dark grey), soft solids (light grey), LC coacervates (cyan), ISO coacervates (green) and no phase separation (white). The fractions of cis- and transazoTAB produced under UV or blue light are also indicated (dashed vertical lines). b, Optical microscopy images of DD/trans-azoTAB ISO coacervate droplets prepared at $350 \mathrm{mM} \mathrm{NaCl}$ in the dark, under UV light, and under blue light (after exposure to UV light). Scale bars, $20 \mu \mathrm{m}$. c, Molar fraction of DD strands sequestered in ISO and LC coacervate droplets in the dark, under UV light, and under blue light (after exposure to UV light). Error bars represent standard deviation from three independent experiments. $\mathbf{d}, \mathbf{e}$, Polymerization yield, $p$, calculated from fitted product distributions (see Supplementary Notes 2 and 3) for reactive LC (d, $100 \mathrm{mM} \mathrm{NaCl}+80 \mathrm{mM}$ EDC) and ISO (e, $200 \mathrm{mM} \mathrm{NaCl}+80 \mathrm{mM} \mathrm{EDC}$ ) coacervate droplets kept in the dark (black dots) or under constant UV light (purple dots), or exposed to cycles of UV and blue light (cyan or green dots). The purple regions highlight times of UV irradiation for samples exposed to UV/blue light cycles. Error bars represent errors associated to uncertainties in the fits used to extract the reaction yield (see Supplementary Note 3). 
Significantly, UV light-mediated dissolution of LC and ISO coacervate droplets resulted in the quantitative release of DD strands in the aqueous solution to give an oligonucleotide concentration of $1.50 \pm 0.04 \mathrm{mg} \mathrm{mL}^{-1}$ (Fig. 4c), so that a 320-fold enhancement of DD local concentration in the darkadapted ISO coacervates compared to the UV-adapted homogeneous solution was observed. Reversibly, exposure to blue light led to the recapture of more than $80 \mathrm{~mol} \%$ of oligonucleotides within the reassembled coacervate phase (Fig. $\mathbf{4 c}$ ). These photoswitchable coacervates thus appeared as a viable platform to reversibly up- or down-regulate the local concentration of oligonucleotides using light, and in turn modulate the ligation reaction.

We therefore proceeded to demonstrate light-actuated ON/OFF oligonucleotide ligation. LC and ISO DDp/trans-azoTAB coacervate droplets prepared under reactive conditions were either stored in the dark for 48 hours, continuously irradiated with UV light, or exposed to cycles of UV and blue light, and polyacrylamide gel scans were performed over time to extract the reaction yield, $p$, and average, $\langle n\rangle$, and maximum, $n_{\max }$, degrees of polymerization (Supplementary Figs. 7 and 18). Results revealed that UV light-exposed dilute DDp/cis-azoTAB samples exhibited a much lower oligonucleotide ligation efficiency compared to dark-adapted samples (Fig. 4d and Supplementary Fig. 7). More specifically, we observed that $p,\langle n\rangle$, and $n_{\max }$ increased monotonously and rapidly for samples kept in the dark, with a stronger increase for LC coacervates compared to ISO droplets, as expected from the enhanced ligation efficiency associated to LC ordering, while UV-exposed samples showed a poor ligation efficiency (Fig. 4d and Supplementary Fig. 19). Strikingly, for samples exposed to cycles of UV and blue light, the reaction yield and degree of polymerization only increased under blue light conditions but remained almost constant during UV light exposure (Fig. 4d and Supplementary Fig. 19). These results therefore demonstrate that oligonucleotide ligation could be periodically modulated with light based on the reversible dissolution of coacervate droplets and the resulting change in the local oligonucleotide concentration.

\section{Conclusion}

In conclusion, we show that charge-balanced mixtures of oligonucleotides and an amphiphilic cationic azobenzene photoswitch produce a rich ensemble of phase-separated complexes ("phenotypes"), including soft solids, LC and ISO coacervate droplets, depending on oligonucleotide sequence and length, azobenzene photoisomerisation and ionic strength. These chemically-enriched phases provide a favourable environment for the non-enzymatic ligation of end-reactive oligonucleotides via carbodiimide chemistry, so that long polynucleotides are produced in situ, while the condensation reaction is only poorly effective in the dilute supernatant. Significantly, polymerization yields are strongly correlated with the nature of the phase in which it occurs, with a markedly increased reaction yield in LC coacervates compared to soft solids or isotropic droplets, indicating that a combination of fluidity and order, typical of liquid crystals, is critical for oligonucleotide ligation. The phase-dependent growth of the DNA population towards longer polymers during ligation ("genotype" variation) further induces structural changes to the compartments themselves ("phenotype" modification), such as the onset of multiphase organization and gradual ISO-to-LC coacervate transition. Such a correlation between compartment structure and in situ reactions offers a promising platform for compartmentcontent coupling in protocells. Since coacervates composed of longer polymers are more stable than those made of shorter ones ${ }^{25}$ and LC coacervates are more stable than ISO ones ${ }^{37} 38$ upon increase in salt and temperature, a positive feedback may also arise, where oligomer elongation stabilizes the compartment, in turn enhancing elongation. Reversible modulation of the assembly and dissolution of coacervate droplets based on light-switchable azobenzene isomerization offers an additional level of temporal modulation over the ligation reaction in periodically changing environmental conditions, which opens perspectives for the sequential incorporation of different oligonucleotides along the synthesized polynucleotide chain as a first step towards the emergence of sequence-defined DNA strands. 
Overall, this work highlights an unexplored approach to the restructuring of coacervate droplets via in situ enzyme-free oligonucleotide elongation, and provides a first step towards the emergence of genotype-phenotype coupling in membrane-free protocells. Combined with selfreplication strategies ${ }^{58}$ and recently reported non-equilibrium coacervate growth-division processes, ${ }^{59}$ such a system could also open perspectives for a systems chemistry realization of Darwinian evolution in protocell populations with synergistic compartment-content reproduction mechanisms. ${ }^{60}$

\section{Materials and Methods}

Materials. Oligonucleotides were purchased from Integrated DNA Technologies as HPLC-purified, freeze-dried, annealed double-stranded oligomers (sequences are given in Supplementary Table 1); sodium chloride $(\mathrm{NaCl})$ and water-soluble 1-ethyl-3-(3-dimethylaminopropyl) carbodiimide hydrochloride (EDC) were purchased from Sigma-Aldrich; SYBR Gold nucleic acid stain was purchased from ThermoFischer Scientific; azoTAB was synthesized using a three-step reaction described previously by azocoupling $p$-ethoxyaniline with phenol, followed by alkylation with dibromoethane then quaternisation with trimethylamine (see reference 41 for details); denaturing polyacrylamide gels were prepared using gel stock solution from Roth; gel running buffer, 1× Tris-Borate Ethylenediaminetetraacetic acid (TBE) buffer, was prepared from 10× TBE from Roth.

Printed circuit board-mounted LEDs operating at $365 \pm 4.5 \mathrm{~nm}$ (model M365D2) or $450 \pm 9 \mathrm{~nm}$ (model M450D3) were purchased from Thorlabs, Inc. and adapted on a custom-made aluminium heat sink. The LEDs were controlled by a T-Cube LED driver that was purchased from Thorlabs, Inc.

Glass coverslips were functionalized with poly(ethylene glycol) brushes to avoid wetting of coacervate droplets. PEGylation was performed by incubation of ethanol-rinsed glass coverslips (0.13$0.17 \mathrm{~mm}$ thick) for 48 hours into a $10 \mathrm{~mL}$ toluene solution containing $500 \mu \mathrm{L}$ of 3[methoxy(polyethyleneoxy)propyl]tri-methoxysilane (90\%, 6-9 PE units, abcr $\mathrm{GmbH}$ ). Coverslips where subsequently rinsed with ethanol and water, and dried with compressed air before being assembled into an observation capillary chamber (as shown in Supplementary Fig. 20).

Preparation of stock solutions. Stock solutions of double-stranded DD, DDp and $\mathrm{DD}_{2}$ oligonucleotides were prepared at $25 \mathrm{mM}$ (nucleobase concentration) by dissolving an appropriate amount of the freeze-dried oligonucleotide powder in nuclease-free Milli- $Q$ water in an Eppendorf tube, and stored at $4^{\circ} \mathrm{C}$. A $25 \mathrm{mM}$ trans-azoTAB aqueous stock solution was prepared by dissolving an appropriate amount of the synthesized azoTAB powder in Milli-Q water in an Eppendorf tube, and the $\mathrm{pH}$ adjusted to 8 with $\mathrm{NaOH}(0.1 \mathrm{M})$ or $\mathrm{HCl}(0.1 \mathrm{M})$. The stock solution was stored in the dark at room temperature before use to ensure complete isomerisation to the trans state, and then protected from light during use by wrapping the tube with aluminium foil. Sodium chloride $(\mathrm{NaCl})$ stock solutions were prepared at various concentrations $(250 \mathrm{mM}, 500 \mathrm{mM}, 1 \mathrm{M}, 2 \mathrm{M}, 4 \mathrm{M})$; and fresh EDC stock solutions were prepared at $400 \mathrm{mM}$ and $1 \mathrm{M}$ concentration just before use.

Equilibrium phase behaviour studies. Complexes between DD and trans-azoTAB were prepared by mixing in the following order aliquots of aqueous stock solutions of trans-azoTAB $(25 \mathrm{mM}, \mathrm{pH} 8), \mathrm{NaCl}$ ( $250 \mathrm{mM}-4 \mathrm{M})$, DD ( $25 \mathrm{mM}$ nucleobase concentration) to reach final concentrations of $5 \mathrm{mM}$ transazoTAB, $5 \mathrm{mM}$ oligonucleotide (nucleobase concentration) and 0-800 $\mathrm{mM} \mathrm{NaCl}$. For studies at varying trans:cis-azoTAB molar ratio, we first mixed a UV-equilibrated cis-azoTAB stock solution $(25 \mathrm{mM}$, kept under UV light for at least 30 minutes) and a dark-adapted trans-azoTAB stock solution (25 $\mathrm{mM}$ ) to produce a mixed trans:cis-azoTAB stock solution $(25 \mathrm{mM}, \mathrm{pH} 8)$ at the desired isomer ratio. This new stock solution was used to prepare samples containing different trans:cis-azoTAB molar ratios. In this case, observations were performed rapidly ( $<30$ minutes) to limit the spontaneous thermal relaxation 
of cis-azoTAB to trans-azoTAB in the dark (half-time of ca. 20 hours ${ }^{45}$ ), and make sure that the trans:cisazoTAB molar ratio did not change during our observations.

Samples were imaged $c a .15$ minutes after formation by loading an aliquot of the suspension onto a custom-made PEG-functionalized capillary chamber. The complexes (aggregates or droplets) were left to settle for $c a$. 5 minutes on the glass coverslip before imaging. Optical microscopy imaging was performed on an inverted Leica DMI 4000B microscope using a $\times 63$ oil immersion objective (HCX PL APO, 1.4 NA) and on an inverted Nikon Ti-U polarized microscope using a $\times 20$ or $\times 50$ objectives. UV or blue light irradiation was performed in situ on the optical microscope by using a CoolLED light source operating at $365 \mathrm{~nm}$ (UV) or $450 \mathrm{~nm}$ (blue), or a Nikon epifluorescence mercury lamp (OSRAM HBO $103 \mathrm{~W} / 2$ ) and Nikon DAPI and FITC filter cubes for UV and blue light, respectively.

Turbidity measurements were also used to characterize the salt- and light-dependent phase behaviour of DD and azoTAB. Here, freshly made samples were added to a 384-well plate and incubated for ca. 5 minutes, then the absorbance at $700 \mathrm{~nm}$ was measured and a reference value (corresponding to $25 \mu \mathrm{L}$ of pure water) subtracted. Cycles of UV and blue light were performed by irradiating the samples for 15 min using alternating UV and blue LED (Thorlabs, Inc): the samples were irradiated for 15 min under UV light using a LED operating at $365 \mathrm{~nm}$, then the absorbance was measured and the samples were irradiated for $15 \mathrm{~min}$ under blue light with a LED operating at $450 \mathrm{~nm}$, and the turbidity monitored at $700 \mathrm{~nm}$ after each irradiation.

Fraction of oligonucleotides in dense complexes vs. supernatant. The fraction of DD sequestered in LC and ISO coacervates vs. supernatant was measured by UV-vis spectroscopy after separation of azoTAB and DD by size exclusion chromatography. Typically, $30 \mu \mathrm{L}$ of a suspension of trans-azoTAB/DD coacervate droplets was produced in an Eppendorf tube $(5 \mathrm{mM}$ nucleobase concentration, $5 \mathrm{mM}$ trans-azoTAB, 200 or $350 \mathrm{mM} \mathrm{NaCl}$ for LC or ISO coacervates, respectively), and separated in three 10 $\mu \mathrm{L}$-aliquots. One aliquot was equilibrated in the dark (10 $\mathrm{min})$, another one was irradiated with UV light (10 $\mathrm{min})$, and the last one was first exposed to UV light $(10 \mathrm{~min}$ ) followed by blue-light irradiation (10 min). Each sample was then centrifuged $(10 \mathrm{~min}, 10000 \mathrm{rpm})$, and the supernatant collected, supplied with $2 \mu \mathrm{L}$ of $5 \mathrm{M} \mathrm{NaCl}$, and further irradiated with UV light to induce complete dissociation of any potential DD/azoTAB complexes. $10 \mu \mathrm{L}$ of $1 \mathrm{M} \mathrm{NaCl}$ were added to the initial tubes after supernatant removal (we observed that these tubes contained a sedimented bulk coacervate phase, for dark and blue light conditions), and the samples were further irradiated under UV light to induce complete dissociation of DD and cis-azoTAB. All samples were added to a PD10 desalting column (GE Healthcare) equilibrated with Milli-Q water to separate azoTAB and DD. Fractions containing only DD were collected and the concentration of oligonucleotide determined in each of them by UV-vis spectroscopy at $260 \mathrm{~nm}$, using an extinction coefficient of $189,089 \mathrm{~L} \mathrm{~mol}^{-1} \mathrm{~cm}^{-1}$ for double stranded DD. ${ }^{61}$ The total amount of DD in bulk coacervate phases and supernatant were determined from the concentration of DD in each fraction and the volume of the fractions (measured by pipetting).

Non-enzymatic oligonucleotide ligation conditions. Typical oligonucleotide ligation experiments were performed in non-binding treated plastic surface 384-well plates (Corning 3544) containing $10 \mu \mathrm{L}$ of reaction solutions: $5 \mathrm{mM}$ trans-azoTAB, $0.42 \mathrm{mM}$ 3'-phosphate DNA 12-mer, DDp (corresponding to 5 $\mathrm{mM}$ negative charge concentration), $80 \mathrm{mM} \mathrm{EDC}$, and 0,100 or $200 \mathrm{mM} \mathrm{NaCl}$ for soft solids, LC and ISO coacervates formation, respectively. To prevent evaporation, samples were covered with $20 \mu \mathrm{L}$ of mineral oil light (Sigma-Aldrich) and well plates were sealed with adhesive PCR plate film (Biorad). UV or blue light irradiation was performed in situ on a Nikon Ti-U inverted optical microscope equipped with a $\times 2$ objective, allowing the illumination of the full well area, by using a Nikon epifluorescence mercury lamp (OSRAM HBO $103 \mathrm{~W} / 2$ ) and Nikon DAPI and FITC filter cubes for UV and blue light, respectively. Reactions were stopped by addition of $2 \mu \mathrm{L} 4 \mathrm{M} \mathrm{NaCl}$, to cause coacervates disassembly, and $20 \mu \mathrm{L}$ ethanolamine $(50 \mathrm{mM}$ stock solution in water). For different reaction times and comparison between UV/blue irradiation and dark conditions, samples prepared from the same initial solution were incubated in different wells and stopped at decided time intervals. Experiments aimed at comparing the ligation products in coacervate phase separately from supernatant were performed in 
$0.2 \mathrm{~mL}$ plastic tubes (Eppendorf), followed by $10 \mathrm{~min}$ centrifugation at $5000 \mathrm{rcf}$ to ensure dense phase sedimentation. Then $9 \mu \mathrm{L}$ of supernatant were extracted by pipetting and transferred to $11 \mu \mathrm{L}$ stop solution, while the remaining $1 \mu \mathrm{L}$ at the bottom of the tube was diluted with $11 \mu \mathrm{L}$ of stop solution.

Polyacrylamide gel electrophoresis. Ligation products analysis were performed using $15 \%$ denaturing polyacrylamide gels ( $20 \mathrm{~cm}$ wide $\times 16 \mathrm{~cm}$ high) containing $8.3 \mathrm{M}$ urea and run in $1 \times$ TBE for 3 hours at constant power of $32 \mathrm{~W}$. Gels were stained with $1 \times$ SYBR Gold for 20 minutes with gentle agitation. Ladders used in gels were composed by an equal mass mixture of 12-, 24-, 48- and 96-mer DNA sequences, corresponding to 1-, 2-, 4- and 8-times repetitions of the DD sequence, respectively.

Gel images analysis was carried out with ImageJ software using a custom MATLAB (MathWorks) script. Estimations of the ligation products distributions from gel bands intensities were performed by measuring the profile peaks areas, normalized on the sum of the area of the peaks. Calculations of reactions yield were obtained by fitting the data with the cumulative products distribution obtained from Flory's theory for simple polymerization ${ }^{49}$ (see Supplementary Notes 2 and 3).

Sample imaging during ligation. Reactive samples were prepared by mixing $5 \mathrm{mM}$ trans-azoTAB, 0.42 $\mathrm{mM}$ 3'-phosphate DNA 12-mer DDp (corresponding to $5 \mathrm{mM}$ negative charge concentration) supplemented with 1× SYBR Gold, $80 \mathrm{mM} \mathrm{EDC,} \mathrm{and} \mathrm{0,} 100$ or $200 \mathrm{mM} \mathrm{NaCl}$ for soft solids, LC and ISO coacervates formation, respectively. Samples were loaded in a custom-made capillary chamber (Supplementary Fig. 20) that was sealed with UV-curing glue (while protecting the samples from UV light exposure using foil). No evaporation was observed in the sealed chamber for up to 2 weeks. Samples were imaged at different times during the reaction on an inverted Leica DMI 4000B microscope using a $\times 63$ oil immersion objective (HCX PL APO, 1.4 NA). Confocal fluorescence microscopy imaging was performed on an inverted Leica DMI RE2 scanning laser confocal microscope.

\section{Author contributions}

T.P.F. and N.M. designed and performed the experiments, undertook data analysis and wrote the manuscript.

\section{Conflicts of interest}

The authors declare no conflict of interest.

\section{Acknowledgements}

We thank D. Cuvelier for the use of his microscope during parts of this study, P. Nghe laboratory at ESPCI for support for gel electrophoresis and M. Todisco for useful discussions. We are indebted to J.P. Douliez, J.-C. Baret and A. Griffiths for critical reading of the manuscript and stimulating discussions. T.P.F. is supported by a Junior Research Chair fellowship from IPGG (Laboratoire d'Excellence, "Investissement d'avenir" program ANR-10-IDEX-0001-02 PSL and ANR-10-LABX-31), ANR-10-EQPX-34 and IPGG AAP High Risk2020 grant. N.M. acknowledges funding from IdEx Bordeaux (ANR-10-IDEX-0302), an "Investissement d'Avenir" program of the French government, managed by the Agence Nationale de la Recherche, and from Région Nouvelle-Aquitaine.

\section{References}

\footnotetext{
${ }^{1}$ Orgel, L.E. Molecular replication, Nature, 358, 203-209 (1992)

${ }^{2}$ Rajamani, S., Vlassov, A., Benner, S., Coombs, A., Olasagasti, F., Deamer, D. Lipid-assisted synthesis of RNA-like polymers from mononucleotides, Orig. Life Evol. Biosph., 38, 57-74 (2008)

${ }^{3}$ Sponer, J.E. et al. Nonenzymatic, template-free polymerization of 3',5' cyclic guanosine monophosphate on mineral surfaces. ChemSystemsChem, 3, e2100017 (2021)
} 
${ }^{4}$ Mast, C.B., Schink, S., Gerland, U., Braun, D. Escalation of polymerization in a thermal gradient, Proc. Nat. Acad. Sci. USA, 110, 8030-8035 (2013)

${ }^{5}$ Ferris, J.P., Hill Jr, A.R., Liu, R., Orgel, L.E. Synthesis of long prebiotic oligomers on mineral surfaces, Nature, 381, 59-61 (1996)

${ }^{6}$ Attwater, J., Wochner, A., Pinheiro, V.B., Coulson, A., Holliger, P. Ice as a protocellular medium for RNA replication. Nat. Commun., 1, 76 (2010)

${ }^{7}$ Monnard, P.A., Kanavarioti, A., Deamer, D.W. Eutectic phase polymerization of activated ribonucleotide mixtures yields quasi-equimolar incorporation of purine and pyrimidine nucleobases. J. Am. Chem. Soc., 125, 13734-13740 (2003)

${ }^{8}$ Adamala, K., Szostak, J.W., Non-enzymatic template-directed RNA synthesis inside model protocells. Science, 342, 1098-1100 (2013)

${ }^{9}$ Szostak, J.W., Bartel, D.P., Luisi, P.L. Synthesizing Life. Nature, 409, 387-390 (2001)

${ }^{10}$ Kurihara, K., Tamura, M., Shohda, K., Toyota, T., Suzuki, K., Sugawara, T. Self-reproduction of supramolecular giant vesicles combined with the amplification of encapsulated DNA. Nat. Chem., 3, 775-781 (2011)

${ }^{11}$ Koga, S., Williams, D.S., Perriman, A.W., Mann, S. Peptide-nucleotide microdroplets as a step towards a membrane-free protocell model, Nat. Chem., 3, 720-724 (2011)

${ }^{12}$ Pir Cakmak, F., Choi, S., Meyer, M., Bevilacqua, P., Keating, C. Prebiotically-relevant low polyion multivalency can improve functionality of membraneless compartments. Nat. Commun. 11, 5949 (2020)

${ }^{13}$ Martin, N. Dynamic synthetic cells based on liquid-liquid phase separation. ChemBioChem, 20, 2553-2568 (2019)

${ }^{14}$ Ghosh, B., Bose, R., Tang, T.-Y.D., Can coacervation unify disparate hypotheses in the origin of cellular life? Curr. Opin. Colloid Int. Sci., 52, 101415 (2021)

${ }^{15}$ Martin, N., Douliez, J.-P., Fatty acid vesicles and coacervates as model prebiotic protocells. ChemSystemsChem, 3, e2100024 (2021)

${ }^{16}$ Martin, N., Li, M., Mann, S. Selective uptake and refolding of globular proteins in coacervate microdroplets. Langmuir, 32, 5881-5889 (2016)

${ }^{17}$ Beneyton, T., Love, C., Girault, M., Tang, D.T.-Y., Baret, J.-C. High-throughput synthesis and screening of functional coacervates using microfluidics. ChemSystemsChem, 2, e2000022 (2020)

${ }_{18}$ Drobot, B., Iglesias-Artola, J.M., Le Vay, K., Mayr, V., Kar, M., Kreysing, M., Mutschler, H., Tang, T.-Y.D. Compartmentalised RNA catalysis in membrane-free coacervate protocells. Nat. Commun., 9, 3643 (2018)

${ }^{19}$ Poudyal, R.R., Guth-Metzler, R.M., Veenis, A.J., Frankel, E.A., Keating, C.D., Bevilacqua, P.C. Template-directed RNA polymerization and enhanced ribozyme catalysis inside membraneless compartments formed by coacervates. Nat. Commun. 10, 490 (2019)

${ }^{20}$ Tang, T.-Y., D., Rohaida Che Hak, C., Thompson, A.J., Kuimova, M.K., Williams, D.S., Perriman, A.W., Mann, S. Fatty acid membrane assembly on coacervate microdroplets as a step towards a hybrid protocell model. Nat. Chem., 6, 527-533 (2014)

${ }^{21}$ Pir Cakmak, F., Marianelli, A.M., Keating, C.D., Phospholipid membrane formation templated by coacervate droplets, Langmuir, 37, 10366-10375 (2021)

${ }^{22}$ Zhang, Y. et al., Giant coacervate vesicles as an integrated approach to cytomimetic modelling, J. Am. Chem. Soc., 143, 2866-2874 (2021)

${ }^{23}$ Lu, T., Spruijt, E. Multiphase complex coacervate droplets. J. Am. Chem. Soc., 142, 2905-2914 (2020)

${ }^{24}$ Mountain, G.A., Keating, C.D. Formation of multiphase complex coacervates and partitioning of biomolecules within them. Biomacromolecules, 21, 630-640 (2020)

${ }^{25}$ Spruijt, E., Westphal, A.H., Borst, J.W., Cohen Stuart, M.A., van der Gucht, J. Binodal compositions of polyelectrolyte complexes. Macromolecules, 43, 6476-6484 (2010)

${ }^{26}$ Vieregg, J.R., Lueckheide, M., Marciel, A.B., Leon, L., Bologna, A.J., Riviera, J.R., Tirrell, M.V. Oligonucleotidepeptide complexes: phase control by hybridization. J. Am. Chem. Soc., 140, 1632-1638 (2018)

${ }^{27}$ Aumiller Jr, W.M., Keating, C.D., Phosphorylation-mediated RNA/peptide complex coacervation as a model for intracellular liquid organelles. Nat. Chem., 8, 129-137 (2016)

${ }^{28}$ Nakashima, K.K., Baaij, J.F., Spruijt, E. Reversible generation of coacervate droplets in an enzymatic network. Soft Matter, 14, 361-367 (2018)

${ }^{29}$ Spoelstra, W.K., van der Sluis, E.O., Dogterom, M., Reese, L. Nonspherical coacervate shapes in an enzymedriven active system. Langmuir, 36, 1956-1964 (2020)

${ }^{30}$ Karoui, H., Seck, M.J., Martin, N. Self-programmed enzyme phase separation and multiphase coacervate droplet organization. Chem. Sci., 12, 2794-2802 (2021)

${ }^{31}$ Donau, C. et al., Active coacervate droplets as a model for membraneless organelles and protocells. Nat. Commun., 11, 5167 (2020) 
${ }^{32}$ Späth, F. et al., Molecular design of chemically fueled peptide-polyelectrolyte coacervate-based assemblies. J. Am. Chem. Soc., 143, 4782-4789 (2021)

${ }^{33}$ Nakata, M., Zanchetta, G., Chapman, B.D., Jones, C.D., Cross, J.O., Pindak, R., Bellini, T., Clark, N.A. End-to-end stacking and liquid crystal condensation of 6-to 20-base pair DNA duplexes. Science, 318, 1276-1279 (2007)

${ }^{34}$ Fraccia, T.P., Smith, G.P., Bethge, L., Zanchetta, G., Nava, G., Klussmann, S., Clark, N.A., Bellini, T. Liquid crystal ordering and isotropic gelation in solutions of four-base-long DNA oligomers. ACS Nano, 10, 8508-8516 (2016)

${ }^{35}$ Fraccia, T.P., Smith, G.P., Zanchetta, G., Paraboschi, E., Yi, Y., Walba, D.M., Dieci, G., Clark, N. A., Bellini, T., Abiotic ligation of DNA oligomers templated by their liquid crystal ordering. Nat. Commun. 6, 6424 (2015)

${ }^{36}$ Todisco, M., Fraccia, T.P. et al., Nonenzymatic polymerization into long linear RNA templated by liquid crystal self-assembly. ACS Nano, 12, 9750-9762 (2018)

${ }^{37}$ Fraccia, T.P., Jia, T.Z. Liquid crystal coacervates composed of short double-stranded DNA and cationic peptides. ACS Nano, 14, 15071-15082 (2020)

${ }^{38} \mathrm{Jia}$, T.Z., Fraccia, T.P. Liquid crystal peptide/DNA coacervates in the context of prebiotic molecular evolution. Crystals, 10, 964 (2020)

${ }^{39}$ Shakya, A., King, J.T. DNA local-flexibility-dependent assembly of phase-separated liquid droplets. Biophys. J., 115, 1840-1847 (2018)

40 Martin, N., Tian, L., Spencer, D., Coutable-Pennarun, A., Anderson, J.L.R., Mann, S. Photoswitchable phase separation and oligonucleotide trafficking in DNA coacervate microdroplets. Angew. Chem. Int. Ed., 58, 1459414598 (2019)

${ }^{41}$ Lafon, S., Martin, N. Reversible photocontrol of DNA coacervation. Methods Enzymol., 646, 329-351 (2021)

${ }^{42}$ Smith, G. P., Fraccia, T. P., Todisco, M., Zanchetta, G., Zhu, C., Hayden, E., Bellini, T., Clark, N. A. Backbone-free duplex-stacked monomer nucleic acids exhibiting Watson-Crick selectivity. Proc. Natl. Acad. Sci. U.S.A. 115, E7658-E7664 (2018).

${ }^{43}$ Wang, Q., Schlenoff, J.B. The polyelectrolyte complex/coacervate continuum. Macromolecules, 47, 3108-3116 (2014)

${ }^{44}$ Rumyantsev, A. M., de Pablo, J. J. Liquid crystalline and isotropic coacervates of semiflexible polyanions and flexible polycations. Macromolecules, 52, 5140-5156 (2019).

${ }^{45}$ Martin, N. et al. Light-induced dynamic shaping and self-division of multipodal polyelectrolyte-surfactant microarchitectures via azobenzene photomechanics. Sci. Rep. 7, 41327 (2017)

${ }^{46}$ De Michele, C., Rovigatti, L., Bellini, T., Sciortino, F. Self-assembly of short DNA duplexes: from a coarse-grained model to experiments through a theoretical link. Soft Matter, 8, 8388-8398 (2012)

${ }^{47}$ Obianyor, C., Newmann, G., Clifton, B.E., Grover, M.A., Hud, N.V., Towards efficient nonenzymatic DNA ligation: comparing key parameters for maximizing ligation rates and yields with carbodiimide activation. ChemBioChem, 21, 3359-3370 (2020)

${ }^{48}$ Maniatis, T., Jeffrey, A., Van deSande, H. Chain length determination of small double- and single-stranded DNA molecules by polyacrylamide gel electrophoresis. Biochemistry 14, 3787-3794 (1975)

${ }^{49}$ Flory, J. Molecular size distribution in linear condensation polymers. J. Am. Chem. Soc. 1, 1877-1885 (1936)

${ }^{50}$ Mangalath, S., Karunakaran, S.C., Newnam, G., Schuster, G.B., Hud, N.V. Supramolecular assembly-enabled homochiral polymerization of short $(\mathrm{dA})_{n}$ oligonucleotides. Chem. Commun., Advance article (2021)

${ }^{51}$ Maffeo, C., Luan, B., Aksimentiev, A. End-to-end attraction of duplex DNA, Nucl. Acid Res., 40, 3812-3821 (2012)

52 Bellini, T. et al., Liquid crystal self-assembly of random-sequence DNA oligomers. Proc. Natl. Acad. Sci. U.S.A., 109, 1110-1115 (2012)

${ }^{53}$ Le Ny, A.-L. M., Lee, C. T. Photoreversible DNA Condensation Using Light-Responsive Surfactants. J. Am. Chem. Soc. 128, 6400-6408 (2006)

${ }^{54}$ Sollogoub, M., et al. Photocontrol of Single-Chain DNA Conformation in Cell-Mimicking Microcompartments. ChemBioChem 9, 1201-1206 (2008)

55 Diguet, A., Mani, N. K., Geoffroy, M., Sollogoub, M., Baigl, D. Photosensitive surfactants with various hydrophobic tail lengths for the photocontrol of genomic DNA conformation with improved efficiency. Chem. Eur. J. 16, 11890-11896 (2010)

${ }^{56}$ Estévez-Torres, A., Baigl, D. DNA compaction: fundamentals and applications. Soft Matter, 7, 6746-6756 (2011)

${ }^{57}$ Hidaka, T., Wee, W.A., Yum, J.H., Sugiyama, H., Park, S. Photo-controllable phase transition of arylazopyrazoleconjugated oligonucleotides, Bioconj. Chem., 32, 2129-2133 (2021)

${ }^{58}$ Adamski, P., Eleveld, M., Sood, A., Kun, A., Szilagyi, A., Czaran, T., Szathmary, E., Otto, S. From self-replication to replicator systems en route to de novo life. Nat. Chem., 4, 389-403 (2020)

${ }^{59}$ A. laneselli, D. Tetiker, J. Stein, A. Kühnlein, C.B. Mast, D. Braun, T.-Y.D. Tang, Non-equilibrium conditions inside rock pores drive fission, maintenance and selection of coacervate protocells. Nat. Chem., early view (2021) 
${ }^{60}$ Matsumura, S., Kun, Á., Ryckelynck, M., Coldren, F., Szilágyi, A., Jossinet, F., Rick, C., Nghe, P., Szathmáry, E., Griffiths, A.D. Transient compartmentalization maintains catalytically active RNA replicators and prevents functional collapse due to parasites. Science, 354, 1293-1296 (2016)

${ }^{61}$ Tataurov, A. V., You, Y., Owczarzy, R. Predicting ultraviolet spectrum of single stranded and double stranded deoxyribonucleic acids. Biophys. Chem., 133, 66-70 (2008) 